\title{
ReaR

\section{Cirugía de implantación de microestimulador del ganglio esfenopalatino. Manejo anestésico y revisión de la literatura}

Ramos Jiménez MD, Santos Martín L, Cordero Tous N, De la Linde Valverde CM

Hospital Universitario Virgen de las Nieves. Granada.

\section{Resumen}

La cefalea en racimos es un tipo de cefalea tigémino-autonómica con síntomas marcadamente disfuncionales debidos al intenso dolor y a la aparición de signos disautonómicos hemicraneales ipsilaterales recurrentes. El tratamiento médico no siempre resulta eficaz o puede no tolerarse debido a sus efectos adversos. El desarrollo de técnicas quirúrgicas que inciden en el tratamiento a través de la estimulación de estructuras directamente relacionadas con el dolor, supone una nueva y esperanzadora herramienta terapéutica para este tipo de pacientes. Además de una cirugía correcta, el tipo de anestesia para la intervención debe ser cuidadosa y adecuarse a la necesidad de trabajar sobre un campo quirúrgico oro-facial amplio. Presentamos un caso de cefalea en racimos de evolución crónica y refractaria a tratamiento, sometido a implantación quirúrgica de un microestimulador en el ganglio esfenopalatino bajo anestesia general.

\section{Introducción}

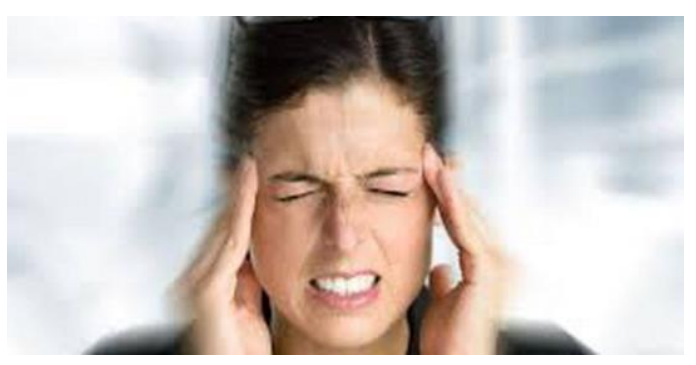

La cefalea en racimos es un tipo de cefalea tigémino-autonómica con síntomas marcadamente disfuncionales debidos al intenso dolor y a la aparición de signos disautonómicos

hemicraneales ipsilaterales recurrentes.

El tratamiento médico no siempre resulta eficaz o puede no tolerarse debido a sus efectos adversos. El desarrollo de técnicas quirúrgicas que inciden en el tratamiento a través de la estimulación de estructuras directamente relacionadas con el dolor, supone una nueva y esperanzadora herramienta terapéutica para este tipo de pacientes. Además de una cirugía correcta, el tipo de anestesia para la intervención debe ser cuidadosa $y$ adecuarse a la necesidad de trabajar sobre un campo quirúrgico oro-facial amplio. Presentamos un caso de cefalea en racimos de evolución crónica $\mathrm{y}$ refractaria a tratamiento, sometido a implantación quirúrgica de un microestimulador en el ganglio esfenopalatino bajo anestesia general.

\section{Introducción}

La cefalea en racimos (CR) es una entidad clínica de naturaleza idiopática caracterizada por crisis de intenso dolor periocularestrictamente unilateral, con signos disautonómicos locales ipsilaterales debidos a una activación parasimpática con hipoactividad simpática. Los ataques suelen seguir un ritmo estacional y circadiano, siendo su 
fisiopatología todavía motivo de estudio, dada la participación de múltiples estructuras como los núcleos trigeminal, salivar superior o el hipotálamo.

La CR es una enfermedad típica del varón joven, con una relación hombre/mujer de 4,3/1 y una edad de comienzo entre la $2^{\mathrm{a}}$ y $5^{\mathrm{a}}$ década $^{1}$. El $80 \%$ de las CR se presentan de forma episódica. La epidemiología completa se expone en la figura 1.

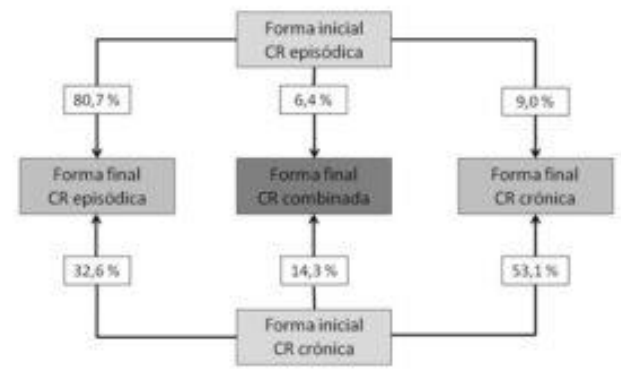

Figura 1. Evolución de las variedades de CR, de acuerdo con Granella et al 2.

El tratamiento inicial es médico, con una vertiente sintomática en la que los fármacos más eficaces son algunos triptanes, los ergóticos y la oxigenoterapia, y un tratamiento basal que suele hacerse con verapamilo, valproato, topiramato, gabapentina $\mathrm{o}$ litio $^{3}$.

La proporción de $\mathrm{CR}$ refractaria a tratamiento oscila entre el $10-39 \%$ según las series ${ }^{4}$. El manejo de las formas crónicas es complejo. En todos los casos, se trata de pacientes de difícil manejo con altísimas tasas de disfuncionalidad, por lo que se deben plantear tratamientos de rescate, habitualmente neuroquirúrgicos ${ }^{5}$.

Los métodos quirúrgicos empleados a lo largo de los últimos 50 años incluyen técnicas lesionales del nervio trigémino o sobre las eferencias parasimpáticas vía nervio facial, nervios petrosos, $\mathrm{y}$ ganglio esfenopalatino (GEP). Sin embargo, parecen ganar terreno en los últimos años las técnicas de estimulación nerviosa, con mejores resultados globales y menor morbilidad asociada, entre ellas destacan la estimulación cerebral profunda a nivel de hipotálamo, la estimulación de los nervios occipitales, y la más reciente, la estimulación del GEP ${ }^{6}$.

Presentamos un caso clínico de CR crónica sometido a la implantación quirúrgica de un microestimulador de GEP bajo anestesia general.

\section{Caso Clínico}

Varón de 55 años con cefalea de 10 años de evolución compatible con $\mathrm{CR}$ del lado derecho de inicio episódico y posterior evolución crónica.

Como antecedentes personales destaca hipercolesterolemia, enfermedad pulmonar obstructiva crónica (EPOC) y tabaquismo importante. Intervenido en dos ocasiones para colocación de estimuladores de ambos nervios occipitales bajo anestesia general, sin complicaciones. No alergias médicas.

En tratamiento actual con verapamilo y melatonina, usando sumatriptán inyectable y oxigenoterapia en las crisis agudas. Tratamiento previo con otros fármacos como sales de litio, gabapentina o topiramato, con mala tolerancia y/o escasos efectos.

El paciente porta un sistema de estimulación nerviosa periférica a nivel de ambos nervios occipitales implantado en 2010, con respuesta discreta, siendo candidato a otras alternativas terapéuticas, en este caso la implantación de un neuroestimulador en el ganglio esfenopalatino derecho, que acepta. 


\section{Manejo Anestésico}

\section{Preoperatorio:}

Durante la visita preanestésica, se recogen los antecedentes personales del paciente previamente referidos. Exploración de vía aérea con Mallampati III, boca séptica, presencia de barba, distancia tiromentoniana y extensión cervical normales. Resto de exploración física normal.

Se indican las pautas habituales de ayuno y premedicación con omeprazol $20 \mathrm{mg}$ y bromazepam 1,5 mg vía oral.

\section{Intraoperatorio:}

Tras comprobar la identidad del paciente, información del preoperatorio y realización de ayuno, pasa a quirófano. Se canaliza una vía periférica $18 \mathrm{G}$ y se monitoriza al paciente con ECG, pulxiometría, tensión arterial no invasiva y BIS. Se administran $2 \mathrm{mg}$ de midazolam iv como premedicación. Se infunden $2 \mathrm{~g}$ cefazolina como profilaxis antibiótica y $4 \mathrm{mg}$ de dexametasona como inicio de terapia esteroidea preventiva de la CR.

Tras preoxigenación, se realiza anestesia general tipo TIVA. Se realiza la inducción con bolos de propofol a 2 $\mathrm{mg} / \mathrm{kg}$, fentanilo a $2 \mathrm{mcg} / \mathrm{kg}$ y rocuronio a $0.6 \mathrm{mg} / \mathrm{kg}$. Paciente ventilable con necesidad de cánula de Guedel por la presencia de barba.

Se realiza intubación orotraqueal con tubo anillado del número 7.5 al segundo intento (en un primer intento, se opta por laringoscopia directa resultando un Cormack-Lehane III; en un segundo intento, se usa Airtraq para la intubación con visión completa de las cuerdas vocales, por lo que la misma es posible). La fijación del tubo se efectúa con seda a nivel de la encía maxilar izquierda una vez se evidencia capnografía (no es posible el uso de venda por indicación de Neurocirugía y la fijación con espadrapo no es una opción debido a la presencia de barba). Ver figura 2.

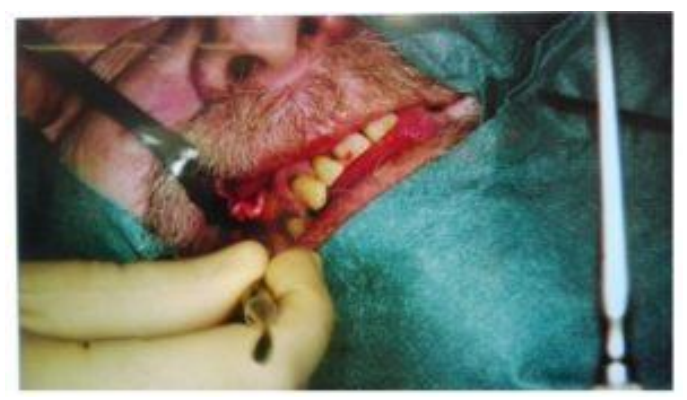

Figura 2. Abordaje transoral a través de la arcada maxilar derecha.

Una vez fijado el tubo y conectado al paciente a ventilación mecánica, se inician las perfusiones continuas de propofol a $5 \mathrm{mg} / \mathrm{kg} / \mathrm{h}$, remifentanilo a $0.05-0.10 \mathrm{mcg} / \mathrm{kg} / \mathrm{min}$ y rocuronio a 5 $\mathrm{mcg} / \mathrm{kg} / \mathrm{min}$.

El abordaje quirúrgico es vía transoral por lo que se hace necesario el taponamiento faríngeo. El paciente se coloca en decúbito supino y de manera neutra. Los ojos del paciente deben ir destapados.

Para la colocación del implante se realiza una incisión a nivel gingival en arcada maxilar derecha y con una disección subperióstica, se consigue llegar a la fosa pterigomaxilar.

Mediante control radioscópico, se coloca el microestimulador del ganglio esfenopalatino (Pulsante ATI Tch) con la punta a nivel del canal vidiano (figura 3). Se realiza TAC craneal intraoperatorio para comprobar la correcta colocación del dispositivo, y tras ello estimulación directa del ganglio esfenopalatino. En este caso, la estimulación dio como resultado un lagrimeo, considerándose adecuada la posición del estimulador. 


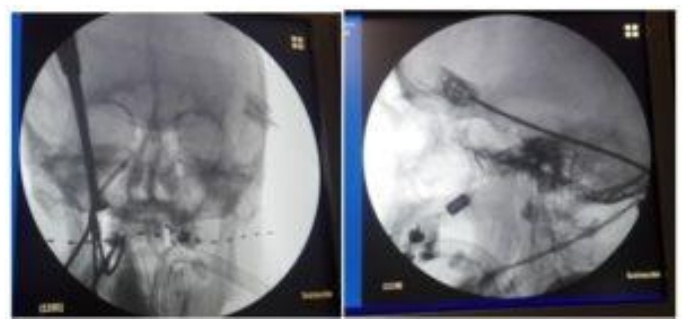

Figura 3. Radiografía intraoperatorias AP y lateral de cráneo. Microestimulador situado en fosa pterigopalatina derecha.

Previo a la educción, se infunde analgesia con $1 \mathrm{~g}$ de paracetamol y 5 mg de cloruro mórfico, así como profilaxis de náuseas $\mathrm{y}$ vómitos postoperatorios con ondansetrón $4 \mathrm{mg}$.

Tras la educción con previa reversión de la relajación neuromuscular con sugammadex a $2 \mathrm{mg} / \mathrm{kg}$ (TOF: 4 respuestas, T4/T1 $>90 \%$ ), se traslada al paciente a Reanimación con buen nivel de conciencia y sin focalidad neurológica aparente.

\section{Discusión}

En la actualidad existe un interés creciente en el desarrollo de técnicas para el control del dolor en pacientes refractarios al tratamiento médico. El auge de las técnicas quirúrgicas percutáneas, cada vez menos invasivas, ha supuesto un avance importante en este sentido. Igualmente, la sustitución de cirugía lesional por procedimientos de neuroestimulación supone, sin duda, una de las líneas de investigación y desarrollo más prometedoras en el ámbito de la terapia del dolor crónico.

La CR es considerada una de las enfermedades dolorosas más limitantes y de más difícil control sintomático ${ }^{7}$. Se cree que el GEP juega un papel importante en el desarrollo $y$ modulación tanto del dolor como de los síntomas autonómicos característicos de la CR. La técnica de tratamiento más novedosa es la estimulación pulsada de GEP mediante un sistema de inserción quirúrgica. Los resultados preliminares del procedimiento indican una tasa de respuesta al dolor en torno al $70 \%{ }^{8}$, resultados similares a la neuroestimulación cerebral profunda o la estimulación de los nervios occipitales, consideradas hasta ahora las más eficaces.

Con respecto al manejo anestésico, es prácticamente inexistente la literatura publicada. Es importante recordar que el campo quirúrgico necesario incluye prácticamente toda la anatomía facial y maxilar de la hemicara sintomática, no solo por la manipulación quirúrgica sino por la necesidad de control radioscópico intraoperatorio, por lo que debe eliminarse cualquier artefacto. El tubo endotraqueal debe fijarse dejando libre el campo quirúrgico, incluyendo las fosas nasales. Como se ha descrito en el caso clínico, la técnica quirúrgica consiste en colocar el microestimulador a través de una incisión en el maxilar superior correspondiente, penetrando hacia la fosa pterigopalatina con un primer disector que ejerce de guía $o$ tutor, controlando su colocación mediante escopia. Tras ello se coloca el microestimulador.

La manipulación del GEP provoca intensas crisis dolorosas intraoperatorias $\mathrm{y}$ estimula las fibras autonómicas, predominantemente las parasimpáticas, suponiendo un momento crítico, ya que pueden desencadenarse rachas de bradicardia en distinto grado e incluso asistolia.

Tras la colocación y comprobación radioscópica de la posición del dispositivo, se estimula el GEP y se evalúan posibles signos autonómicos como el enrojecimiento local, lagrimeo, rinorrea o salivación. $\mathrm{Su}$ aparición indica la correcta estimulación de las fibras autonómicas y, por tanto, un dato más de una adecuada colocación anatómica. 
No existen publicaciones sobre la técnica anestésica más adecuada en el intraoperatorio. En nuestro caso se escogió la anestesia general, tipo TIVA con sistema de infusión manual convencional de propofol y remifentanilo. Esta es la opción habitual en neuroanestesia. Podría ser interesante plantear otras alternativas como, por ejemplo, el uso de dexmedetomidina y paciente despierto, aunque la falta de publicaciones al respecto y la posibilidad de control inadecuado del dolor al estimular el GEP nos hizo descartar esta opción. Es más, la presencia de signos autonómicos objetivos y visibles en la mayor parte de los casos hace innecesario el estado vigil para comprobar la correcta situación del dispositivo.

Durante el postoperatorio inmediato, uno de los problemas más importantes es el control de las crisis de dolor, característicamente agudizadas en las primeras $24 \mathrm{~h}$ tras la manipulación quirúrgica del GEP. Para ello es necesaria la oxigenoterapia a alto flujo y la administración de triptanes o analgésicos opioides. Se debe reintroducir la medicación oral basal del paciente en cuanto se inicie la tolerancia oral para asegurar la continuidad del tratamiento preventivo hasta el inicio de la neuroestimulación.

También es necesario vigilar el posible sangrado a nivel de la encía que se puede controlar mediante compresión o con una gasa con una pequeña dosis de ácido tranexámico. Otros cuidados básicos incluyen aplicar frío local para minimizar la inflamación de la zona maxilar y tejido blando, enjuague bucal con clorhexidina cada $12 \mathrm{~h}$ y pautar antibioterapia durante al menos 3 días más para evitar la infección del material.

Como es evidente, la escasa literatura publicada sobre este procedimiento hace difícil establecer pautas anestésicas concretas más allá de las genéricas que habitualmente se utilizan en el ámbito de la neuroanestesia. Aportamos nuestra experiencia con este caso clínico.

\section{Bibliografía}

- Fischera M, Marziniak M, Gralow I, Ever S. The incidence and prevalence of cluster headache: a meta-analysis of population based studies. 2008;28:614-8. (릴ed)

- Granella F, Micieli G, Zanferrai C, Cavallini A, Manzoni GC, Nappi GC. Natural history of cluster headache: an epidemiological study. Cephalalgia. 1987;7(suppl.6):319-20.

- May A. update on the diagnosis and managet of trigémino-autonomic headaches. J Neurol. 2006;253:1525-32. (ubMed)

- Dodick DW, Capmbell JK. Cluster headache: diagnosis, managment and treatment. En: Silberstein SD, Lipton RB, Dalessio DJ. Wolff's headache and other head pain. $7^{\circ}$ ed. Oxford: Oxford University Press. 2001;283-309. (

- Assaf AT, Hillerup J, Rostgaard M, Puche M, Blessmann $\mathrm{C}$, et al. Technical and surgical aspects of the sphenopalatine ganglion (SPG) microstimulator insertion procedure. J. Oral Maxillofac. Surg. 2016; 45: 245-54. (吕ML)

- M. Asensio Samper. Técnicas intervencionistas para el tratamiento del dolor crónico. Rev Esp Anestesiol Reanim 2012;59:174-6. (HTML)

- Ansarinia M, Rezai A, Tepper SJ, Steiner CP, Stump J, Stanton-Hicks M, et al. Electrical stimulation of sphenopalatine ganglion for acute treatment of cluster headaches. Headache 2010;50:1164-74. (ubMed)

- Schoenen J, Jensen RH, Lanteri-Minet M, Lainez MJ, Gaul C, Goodman AM, et al. Stimulation of the sphenopalatine ganglion (SPG) for cluster headache treatment. Path-way CH-1: a randomized, sham-controlled study.

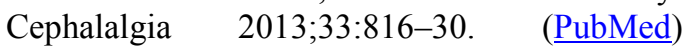
( $\underline{\mathrm{HTML}}$ ( $\underline{\mathrm{PDF}})$ 
Aceptado para el blog en noviembre

María Dolores Ramos Jiménez

lolaramos@hotmail.com

MIR de Anestesiología, Reanimación y Terapéutica del Dolor.

Hospital Universitario Virgen de las Nieves.

Granada. de 2018. 